\title{
The Geometry of Singular Foci of Planar Linkages
}

\author{
Charles W. Wampler \\ General Motors Research Laboratories, Mail Code 480-106-359, \\ 30500 Mound Road, Warren, MI 48090-9055, USA
}

\begin{abstract}
The focal points of a curve traced by a planar linkage capture essential information about the curve. In a previous paper, we showed how to determine the singular foci of planar linkages from an expression for the tracing curve derived by use of the Dixon determinant. This paper gives an alternative approach to finding the singular foci, one that lends itself to simple geometric interpretations and does not require a derivation of the tracing curve equation. In many cases, singular foci can be determined from a simple graphical construction. The method is demonstrated for one inversion each of the Stephenson-3 six-bar and the Watt-1 six-bar. A by-product of the study is a technique for illustrating the non-real points on a tracing curve. Knowledge of the singular foci will be helpful in further study of path cognates.
\end{abstract}

Key words: Focal points, singular focus, Foci: singular, Linkages: planar, isotropic coordinates

\section{Introduction}

The focal triangle of a planar four-bar is well-known in kinematics: two vertices are the fixed pivots of the four-bar and the third vertex is obtained by noting that the focal triangle is similar to the coupler triangle of the linkage. These three points are the singular foci of the four-bar coupler curve. They play a central role in the determination of path cognates, which are distinct mechanisms that produce the same curve. Roberts' Theorem [11] proved that every four-bar curve is triply generated, and as shown by Cayley [3], the three Roberts cognates obtain from choosing any two of the three singular foci as

Email address: charles.w.wampler@gm.com (Charles W. Wampler). 
the fixed pivots. The uniqueness of Roberts cognates is demonstrated in [12]. (See also [2, pp. 339-341] and [6, pp. 150-155] for more on Roberts cognates.)

It is natural to wonder how these results might generalize to more complicated planar linkages. It is known, for example, that six-bar linkages have fully circular tracing curves (often called coupler curves) of degree either 14, 16, or 18, depending on the type of six-bar [13]. This implies that they have either 7,8 , or 9 singular foci. Is there a simple geometric relation that determines the singular foci of a six-bar, something analogous to the focal triangle of the four-bar? How do the singular foci relate to path cognates for six-bars? This paper gives a method for answering the first question and partially addresses the second. The method applies not just to six-bar linkages, but to any planar linkage.

An algorithm for computing the singular foci of any planar linkage having all rotational joints, with extensibility to prismatic joints as well, is given in [17]. That approach extracts the singular foci from an expression for the tracing curve derived in [16] via the Dixon determinant. This method can be conveniently automated to handle any linkage, but it does not give much insight into the geometry of the foci. One can easily use a numerical implementation of that approach to observe, for example, that the fixed pivots of a mechanism are often singular foci. One can also write out the conditions (preferably with use of computer algebra) to get symbolic expressions. The method of the current paper yields a better understanding of the foci in terms of geometric diagrams and often leads to simple symbolic expressions. The new geometric picture also explains why some singular foci appear with multiplicity greater than one. The drawback of the geometric approach is that it is not easily automated, so that we use it on a case-by-case basis.

The paper proceeds as follows. First, in $\S 2$ we review how singular foci relate to the behavior of a planar curve at infinity. In particular, as shown in [17], it is convenient to formulate equations in isotropic coordinates and to use a two-homogenization of the tracing curve. Before presenting the new method, we first discuss in $\S 3$ a technique for drawing a linkage in a "non-real" configuration, including drawing it as the tracing point reaches infinity. In $\S 4$, we develop the new method for finding singular foci and apply it to a Stephenson-3 six-bar and a Watt-1 six-bar. We conclude with two brief sections: one discusses how singular foci relate to the problem of finding all path cognates for a given linkage $(\S 5)$, and one identifies a class of mechanisms, called "coupler mechanisms," for which the theory is especially easy to apply ( $\S 6)$. 


\section{Background}

Generally speaking, the curves generated by planar linkages have special properties not expected of a general algebraic curve of the same degree. A notable example is the four-bar coupler curve, which is a sixth-degree curve. A general sextic in the plane has 28 terms, while a four-bar with a coupler triangle has only nine link parameters. Clearly a four-bar coupler curve is not a general sextic. Most of the difference is due to the fact that the curve is tricircular, which means that in the usual Cartesian coordinates, the coupler-curve equation is of the form

$$
\begin{aligned}
a_{0}\left(x^{2}+y^{2}\right)^{3} & +\left(a_{1} x+a_{2} y\right)\left(x^{2}+y^{2}\right)^{2} \\
& +\left(a_{3} x^{2}+a_{4} x y+a_{5} y^{2}\right)\left(x^{2}+y^{2}\right)+f(x, y)=0,
\end{aligned}
$$

where $a_{0}, \ldots, a_{5}$ are constant coefficients and $f(x, y)$ is a cubic in $x, y$. This equation has in total only 16 constants, which accounts for the bulk of the difference. ${ }^{1}$ It has also been shown that general six-bar curves are also fully circular [13]: depending on the type of six-bar, they are degree 14, 16, or 18 with circularity 7,8 , or 9 . Thus, circularity is a fundamental property of curves drawn by planar linkages having rotational joints. In the next few paragraphs, we use the four-bar equation to illustrate concepts that generalize naturally to any planar algebraic curve.

A circular curve passes through two special points at infinity, called the isotropic points, denoted $I$ and $J$. As is readily seen from the sixth-degree terms in Eq.(1), as $x$ and $y$ grow large, they must be in the ratio $x / y= \pm \mathrm{i}$, where $\mathrm{i}=\sqrt{-1}$. Or more precisely, we may homogenize Eq.(1) by substituting $(x, y)=(X / W, Y / W)$ and clearing denominators to get

$$
\begin{aligned}
a_{0}\left(X^{2}\right. & \left.+Y^{2}\right)^{3}+W\left(a_{1} X+a_{2} Y\right)\left(X^{2}+Y^{2}\right)^{2} \\
& +W^{2}\left(a_{3} X^{2}+a_{4} X Y+a_{5} Y^{2}\right)\left(X^{2}+Y^{2}\right)+W^{3} F(X, Y, W)=0
\end{aligned}
$$

where $F(X, Y, W)$ is the homogenization of $f(x, y)$. Using the bracket notation $[X, Y, W]$ to indicate that in homogeneous coordinates only the ratio of the coordinates matter, we may write the points where the curve hits infinity, $W=0$, as

$$
I:=[1,-\mathrm{i}, 0] \quad \text { and } \quad J:=[1, \mathrm{i}, 0]
$$

$\overline{1}$ The full accounting is that the curve is also trinodal, meaning it has three double points, and these nodes must lie on the focal circle [3]. 
The four-bar curve passes through each of $I$ and $J$ three times, each time along a different tangent line. The singular foci of any circular curve are the points that are common to two such tangents [4]. This is important enough that we restate it as a formal definition.

Definition $1 A$ singular focal point ${ }^{2}$ of an algebraic curve in the plane is the intersection of a tangent at isotropic point I with a tangent at isotropic point $J$.

It is not immediately clear from this definition why singular foci are of particular interest nor how we would determine them from the coupler-curve equation. This all becomes much easier to fathom if we change our expressions from the Cartesian coordinates $(x, y)$ to isotropic coordinates $(p, \bar{p})$, where

$$
(p, \bar{p})=(x+i y, x-i y)
$$

This is, in effect, just a mapping of the Cartesian plane into the complex (Argand) plane, so that when $x$ and $y$ are real, $p$ is just the corresponding complex vector, and $\bar{p}$ is its complex conjugate. Despite the greater familiarity of Cartesian coordinates, it can be argued that almost all derivations in planar kinematics are more easily developed in isotropic coordinates. Their use in kinematics has a long history, see for example $[1,5,7,11]$. More exposition in the context of recent work can be found in [14-17].

Since $x^{2}+y^{2}=p \bar{p}$, in isotropic coordinates Eq.(1) becomes

$$
a_{0} p^{3} \bar{p}^{3}+\left(b_{1} p+\bar{b}_{1} \bar{p}\right) p^{2} \bar{p}^{2}+\left(b_{2} p^{2}+b_{3} p \bar{p}+\bar{b}_{2} \bar{p}^{2}\right) p \bar{p}+g(p, \bar{p})=0,
$$

where $g(p, \bar{p})$ is a cubic polynomial. Note that if the coefficients in the Cartesian expression are real, then the coefficients on monomials $p^{j} \bar{p}^{k}$ and $p^{k} \bar{p}^{j}$ are complex conjugates of each other, for example, $b_{1}=\left(a_{1}-a_{2} \mathrm{i}\right) / 2$ and $\bar{b}_{1}=\left(a_{1}+a_{2} \mathrm{i}\right) / 2$. This complex conjugate relationship between coefficients holds in general, not just for four-bars.

In isotropic coordinates we have the desirable situation that the circularity of the curve is completely determined by which monomials appear. The salient feature of Eq.(4) is that although it is of sixth degree, variables $p$ and $\bar{p}$ only appear up to degree three; that is, it is a bicubic equation. Defining the bidegree $b$ as the highest power of $p$ that appears, which is the same as the highest power of $\bar{p}$ that appears, and letting $d$ be the total degree of the equation, we say that the difference $c=d-b$ is the circularity of the curve.

$\overline{2}$ Bottema and Roth [2] note that singular foci are also sometimes called special, principal, or Laguerre foci. 
The behavior at infinity of the curve is not altered by a linear change of coordinates. Homogenizing Eq.(4) via the substitution $(p, \bar{p})=(P / W, \bar{P} / W)$, one obtains an expression analogous to Eq.(2) as

$$
\begin{aligned}
a_{0} P^{3} \bar{P}^{3} & +W\left(b_{1} P+\bar{b}_{1} \bar{P}\right) P^{2} \bar{P}^{2} \\
& +W^{2}\left(b_{2} P^{2}+b_{3} P \bar{P}+\bar{b}_{2} \bar{P}^{2}\right) P \bar{P}+W^{3} G_{1}(P, \bar{P}, W)=0
\end{aligned}
$$

where $G_{1}$ is the one-homogenization of $g(p, \bar{p})$. The curve still hits infinity in two triple points, which keep their labels $I$ and $J$, as

$$
I:=[1,0,0] \text { and } J:=[0,1,0] .
$$

The final step in simplifying the whole picture is to consider a two-homogenization of the plane. We introduce a separate homogeneous coordinate for $p$ and $\bar{p}$ by making the substitutions $(p, \bar{p})=(P / V, \bar{P} / \bar{V})$ and clearing denominators. Now the four-bar coupler curve equation reads

$$
\begin{aligned}
a_{0} P^{3} \bar{P}^{3} & +\left(b_{1} P \bar{V}+\bar{b}_{1} \bar{P} V\right) P^{2} \bar{P}^{2} \\
& +\left(b_{2} P^{2} \bar{V}^{2}+b_{3} P \bar{P} V \bar{V}+\bar{b}_{2} \bar{P}^{2} V^{2}\right) P \bar{P}+G_{2}(P, \bar{P}, V, \bar{V})=0,
\end{aligned}
$$

where $G_{2}$ is the two-homogenization of $g$. As discussed in [17], this transformation blows up isotropic points $I$ and $J$ into the lines at infinity $V=0$ and $\bar{V}=0$, respectively. (See also [8, ex.7.22] for a discussion of blowing up from the perspective of algebraic geometry.) This maneuver restructures the picture at infinity, so that the former multiple intersections of the coupler curve with $I$ and $J$ are now separated into (generally) distinct points of intersection with these lines at infinity. More precisely, for each line through $I$ in the one-homogeneous formulation there is a corresponding point along the line $V=0$ in the two-homogeneous formulation. A similar one-to-one correspondence holds for lines through $J$ and points on $\bar{V}=0$.

The bottom line is that a complete picture of the behavior of the coupler curve at infinity is obtained by finding its intersections with the lines $V=0$ and $\bar{V}=0$. But substituting $V=0$ into the coupler curve equation means that all but the terms of top degree in $P$ drop out. Also, since $[P, V]$ are homogeneous coordinates, we may set $P=1$ when $V=0$. The upshot is that we are left with just a homogeneous cubic equation in $\bar{P}, \bar{V}$. This is just the homogenization of the leading terms in $p$ of the original equation. We get a similar result from setting $\bar{V}=0$, but now we are picking out the leading terms in $\bar{p}$. These two leading polynomials have coefficients that are complex conjugates of each other, so the solutions of one are just the complex conjugates of those of the 
other. Hence, we just need to solve one of them to know how the curve hits infinity.

Since the solutions to these leading polynomials correspond to the tangents of the coupler curve at the isotropic points, we have the following result concerning singular foci.

Theorem 1 [17]. Let $F(P, V, \bar{P}, \bar{V})$ be the two homogenization of a polynomial $f(p, \bar{p})$. The singular focal points of $f(p, \bar{p})=0$ are the points $(p, \bar{p})=$ $\left(q_{J}, \bar{q}_{I}\right)$, where $q_{J}$ is any root of $F$ of the form $\left(\left[q_{J}, 1\right],[1,0]\right)$, and $\bar{q}_{I}$ is any root of $F$ of the form $\left([1,0],\left[\bar{q}_{I}, 1\right]\right)$. These are just the roots of the leading polynomials of $f(p, \bar{p})$.

Finally, we see why the singular foci are of interest. They tell us where the curve reaches infinity and they depend only on the terms of highest degree. This is useful in the search for path cognates, because the two cognate mechanisms must reach infinity in the same points, and hence they must have the same singular foci. These points are easier to evaluate than general points of the curve, because they depend only on the leading terms. Another implication is that if two curves have a common singular focus, it means they have points in common on each of the lines $V=0$ and $\bar{V}=0$, and thus the number of finite intersections is reduced by two.

A final remark is necessary to clarify what follows. A four-bar coupler curve has three points on each of the lines $V=0$ and $\bar{V}=0$, so by Thm. 1, there are nine singular foci. However, we have already noted that the three $q_{I}$ and three $q_{J}$ appear as complex conjugates of each other. Thus, the three pairs $\left(q_{J}, q_{I}\right)$ that match up the complex conjugates are "real" points. These are the ones that form the focal triangle of the four-bar, and they completely encode all the focal information. Since we are only interested in real curves, it is always enough to only consider these real focal points, and so just finding the $q_{I}$ is sufficient.

This paper is concerned with finding the singular foci of curves traced out by planar linkages. For any planar linkage with all rotational joints, the singular foci can be found by applying the Dixon determinant to get the tracing curve equations, as in [16], and then picking out the leading polynomials [17]. In this paper, we give a method that skips the first step and directly finds the singular foci from the loop equations for a mechanism. This has the advantage of giving simpler expressions and also giving a simpler geometric interpretation of the singular foci. In fact, we can find many singular foci by drawing simple diagrams that depend on the shape of the links in the mechanism. But first, we must work out a method of drawing a linkage at a non-real configuration. 


\section{Drawing Non-Real Configurations}

By Def.1, the singular foci are determined by how the tracing curve reaches the isotropic points. These points are not on the real portion of the curve and they are at infinity, so at first thought, it would not seem possible to draw the linkage in such a position. This section will reveal a method for drawing non-real points on the tracing curve, which will subsequently be used to show the locations of the singular foci.

The tracing curve, $\mathcal{C} \in \mathbb{C}^{2}$, may be regarded as the projection onto $(p, \bar{p})$ of the motion curve, $\hat{\mathcal{C}} \in \mathbb{C}^{2 N}$, in the variables $(\mathbf{x}, p)=\left(\theta_{1}, \ldots, \theta_{N-1}, p\right)$ and their conjugate variables $(\overline{\mathbf{x}}, \bar{p})=\left(\bar{\theta}_{1}, \ldots, \bar{\theta}_{N-1}, \bar{p}\right)$. The curve $\hat{\mathcal{C}}$ shows the coordinated motion of all the links along the motion, while $\mathcal{C}$ is just the motion of the tracing point. To draw the linkage at a point on $\mathcal{C}$, we must know the placement of all the links, which is the information in the corresponding point of $\hat{\mathcal{C}}$.

Throughout the article, we denote complex conjugation with an asterix, that is, $c^{*}$ is the complex conjugate of $c$.

As shown in [14] and used in [15-17], the curve $\hat{\mathcal{C}}$ is given by $n=N / 2$ primary loop equations and $n$ conjugate equations, written for $k=1, \ldots, n$ as

$$
\begin{aligned}
& c_{k 0}+\sum_{i=1}^{N-1} c_{k i} \theta_{i}+c_{k N} p=0 \\
& c_{k 0}^{*}+\sum_{i=1}^{N-1} c_{k i}^{*} \bar{\theta}_{i}+c_{k N}^{*} \bar{p}=0 .
\end{aligned}
$$

In addition, the joint variables must satisfy the condition of unit magnitude rotation, written

$$
\theta_{i} \bar{\theta}_{i}-1=0, \quad i=1, \ldots, N-1 .
$$

(Those unfamiliar with this formulation may understand it by examining the examples in the next section.) For real points on the curve, $\bar{\theta}_{i}^{*}=\theta_{i}$, all $i$, which is synonymous with $\theta_{i}$ having unit magnitude. The non-real points on the curve are those for which Eqs. $(8-10)$ hold, but $\bar{\theta}_{i}^{*} \neq \theta_{i}$, for some $i$. Then, $\theta_{i}$ and $\bar{\theta}_{i}$ are stretch-rotations, one of which magnifies link $i$ and the other reduces link $i$ by a reciprocal factor.

To illustrate the linkage at a non-real point, we draw two sets of loop-closures: one for the primary loop equations and one for the conjugate loop equations. An example is shown in Fig.1 for a four-bar linkage. For ease of visualization, 


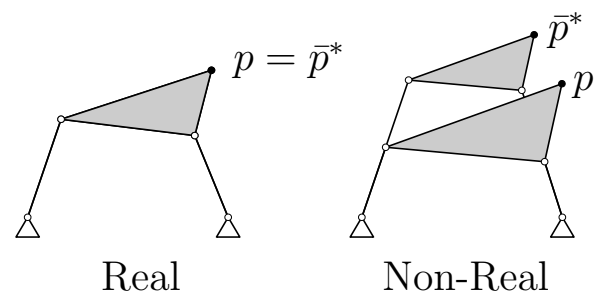

Fig. 1. Real and non-real points on a four-bar coupler curve.

it is helpful to draw the complex-conjugate of the conjugate loop equation, namely

$$
c_{k 0}+\sum_{i=1}^{N-1} c_{k i} \bar{\theta}_{i}^{*}+c_{k N} \bar{p}^{*}=0 .
$$

For real points, the primary and conjugate loops are identical, so they appear as one at the left in Fig.1. But for a non-real point, such as illustrated at the right in Fig.1, one sees that the although link $i$ has the same rotation angle in both the primary loop and the conjugate loop, it is magnified in one and demagnified in the other. Even so, the loop remains closed in both. For the non-real point, we have taken $\theta_{1}$ at the same angle as the real diagram, but its magnitude is decreased from 1 to 0.7 . As the magnitude of $\theta_{1}$ decreases further, the coupler triangle for the primary loop will settle down onto the fixed pivots, while in the conjugate loop, the two binary links grow without bound, taking point $\bar{p}^{*}$ to infinity. The next few paragraphs will make this observation precise.

\section{Diagrams of Singular Foci}

Now that we can draw the linkage in non-real configurations, it is possible also to draw it at a singular focus. The singular foci are the locations of the tracing point $p$ when the conjugate tracing point $\bar{p}$ is at infinity. Generally, there is more than one such point.

It is best to begin by writing the equations that describe the singular foci. By Theorem 1, the singular foci of the tracing curve $\mathcal{C}$ are determined by its two-homogeneous roots at infinity. Thus, one may determine the singular foci of $\mathcal{C}$ as the projections of the roots at infinity of $\hat{\mathcal{C}}$. Let $g(\mathbf{x}, p, \overline{\mathbf{x}}, \bar{p})=0$ denote the entire system of $2 N-1$ equations in $2 N$ variables given by Eqs.(8-10), and let $G(\mathbf{X}, P, V, \overline{\mathbf{X}}, \bar{P}, \bar{V})$ be the two-homogenization of $g$. Accordingly, we wish to find the solutions of the system

$$
G(\mathbf{X}, P, 1, \overline{\mathbf{X}}, 1,0)=0
$$


which when written out is

$$
\begin{aligned}
c_{k 0}+\sum_{i=1}^{N-1} c_{k i} \Theta_{i}+c_{k N} P & =0, \quad k=1, \ldots, n, \\
\sum_{i=1}^{N-1} c_{k i}^{*} \bar{\Theta}_{i}+c_{k N}^{*} & =0, \quad k=1, \ldots, n, \\
\Theta_{i} \bar{\Theta}_{i} & =0, \quad i=1, \ldots, N-1 .
\end{aligned}
$$

This system of equations is simple to solve because Eqs.(12,13) are linear and Eqs.(14) imply either $\Theta_{i}=0$ or $\bar{\Theta}_{i}=0$ for all $i$. Finding all solutions is only a matter of cycling through the choices of $\Theta_{i}=0$ vs. $\bar{\Theta}_{i}=0$. Generally, since the extra variable $P$ appears in Eq.(12) where no corresponding variable appears in Eq. (13), the solutions have $n$ variables among $\left(\Theta_{1}, \ldots, \Theta_{N-1}\right)$ that are zero and the complementary $n-1$ variables among $\left(\bar{\Theta}_{1}, \ldots, \bar{\Theta}_{N-1}\right)$ are zero. This satisfies all of Eqs.(14) and leaves just enough variables to solve the linear systems Eqs. $(12,13)$. An exception to this rule occurs when the linear systems are not both full rank; this is related to the appearance of extraneous factors in the Dixon determinant, as we shall discuss below in a re-examination of the Watt-1 six-bar.

An appealing feature of this approach is that we may interpret the results as simple geometric diagrams. The solutions we seek use one half of the links, including the ground link, to close the primary loop equations, Eq.(12). The remaining links close the conjugate loop equations, Eq.(13). Normally, these two sets of equations are coupled together through the unit magnitude conditions, Eq.(10), but at infinity these conditions are replaced by Eq.(14), which decouples the two subsystems.

In the primary loop closure diagram for Eq.(12), the location of the tracing point is a singular focus. However, one must not neglect to check the existence of a solution in the conjugate loop diagram. In that diagram, the ground link is scaled to zero; that is, all ground pivots move to the origin. Meanwhile, $\bar{P}=1$; that is, the conjugate tracing point is placed off the origin one unit.

The diagrams for the singular foci are limiting cases of the finite tracing curve as the scale of some links shrink to zero in the primary loop diagram while their reciprocals in the conjugate diagram grow infinitely large. We have already observed the beginning of this process for a four-bar in Fig.1. In the limit, the conjugate loop diagram may be understood as a picture of the linkage zoomed out infinitely, so that the finite links shrink to nothing at the origin and only the links that have been infinitely magnified appear.

Some partitions of the links between the two sets of loops may fail to have 


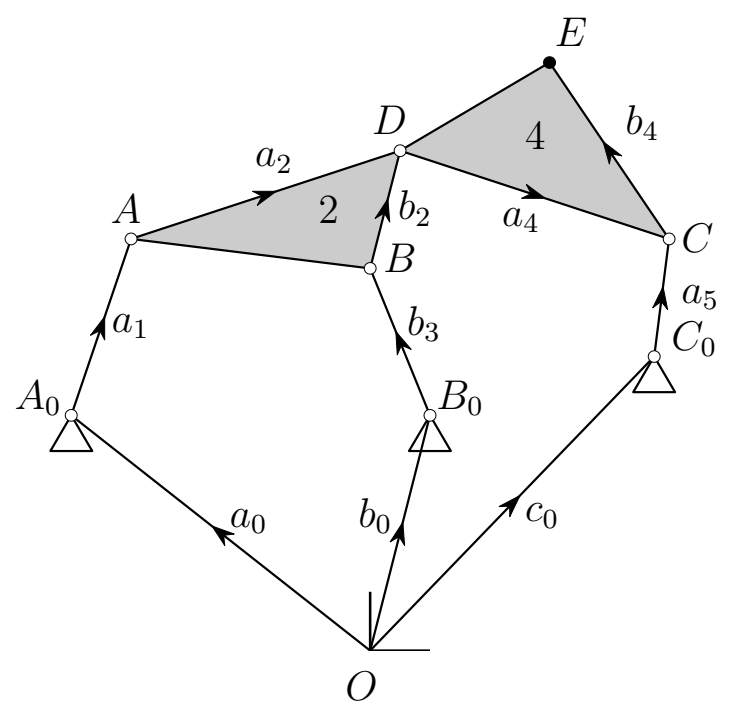

Fig. 2. Four-bar (point $D$ ) and Stephenson-3 six-bar (point $E$ ) path-generating linkages

solutions. On the primary side, this may happen for example when all $\Theta_{i}$ are zero in a loop that includes the (nonzero) ground link. On the conjugate side, this happens when all $\bar{\Theta}_{i}$ are zero on some path between ground and the tracing point.

The best way to understand these diagrams is to draw them for a few examples. We begin with the familiar four-bar linkage.

Example 1 Four-bar focal diagrams. Consider the four-bar linkage $A_{0} A D B B_{0}$, a sub-mechanism of the six-bar in Fig.2. The focal diagrams are shown in Fig.3, with the diagrams for the primary loop closure at the left and the diagrams for the conjugate loop shown on the right. Unlike in Fig.1, here the conjugate loop diagram is separated from the primary loop diagram so that it can be rescaled to finite size. In the main focal diagram, we draw the ground link and a stretch-rotation of one of the three moving links, scaling the other two to zero. The connectivity between links remains the same despite some of them having zero size. In case (a), $\Theta_{1}=\Theta_{2}=0$, binary link 1 and the coupler link 2 are scaled to zero, and the tracing point is at ground pivot $A_{0}$. In case (b), $\Theta_{3}=\Theta_{2}=0$, and the tracing point is at ground pivot $B_{0}$. Finally, in case (c), $\Theta_{1}=\Theta_{3}=0$, the coupler triangle is stretch-rotated to bridge the ground pivots, placing the third singular focal point at $p=a_{0}+a_{2}\left(b_{0}-a_{0}\right) /\left(a_{2}-b_{2}\right)$. In each case, the conjugate loop diagrams are satisfactory, once again confirming the classical result that there are three singular foci, and the focal triangle is similar to the coupler triangle.

The singular foci of a Stephenson-3 mechanism are related to its four-bar submechanism in a special way. One may see from the following example that a similar relationship will hold for any linkage formed by adding a tracer dyad 


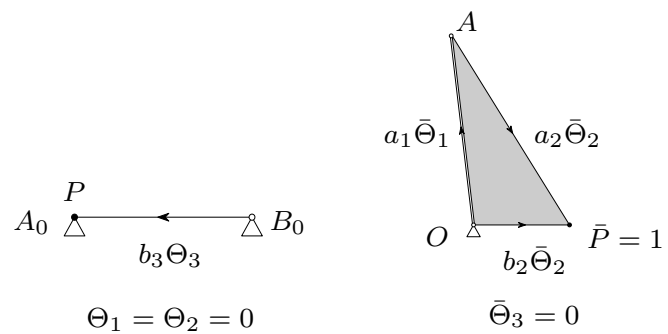

(a)

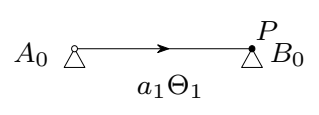

$$
\Theta_{2}=\Theta_{3}=0
$$

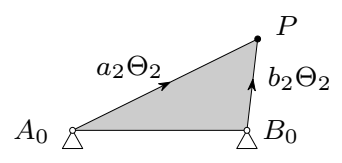

$\Theta_{1}=\Theta_{3}=0$

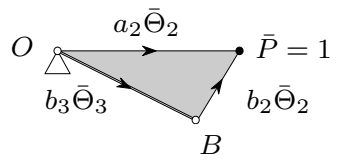

$\bar{\Theta}_{1}=0$

(b)

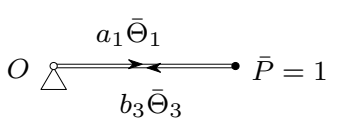

(c)

$\bar{\Theta}_{2}=0$

Fig. 3. Focal diagrams for a four-bar: primary loop on left and conjugate loop on right for each.

to a simpler linkage.

Example 2 Stephenson-3 focal diagrams. Refer again to the Stephenson-3 six-bar linkage shown in Fig.2. This linkage has nine singular foci as diagrammed in Fig.4. This time, to save space, the conjugate loop diagrams are omitted. In the main focal diagram, we draw the ground link and a stretchrotation of two of the five moving links, scaling the other three to zero. The diagrams are arranged in columns, according to whether links 4 and 5 are scaled to zero. When $\Theta_{4}=0$ with $\Theta_{5} \neq 0$, the focal diagrams give singular foci $F_{1}, F_{2}, F_{3}$ that are just the singular foci of the four-bar, as in the previous example, with link 5 closing the loop to ground pivot $C_{0}$. When $\Theta_{4} \neq 0$ and $\Theta_{5}=0$, one obtains a new focal triangle that is a stretch-rotation about $C_{0}$ of the four-bar focal triangle; the stretch-rotation factor is the ratio of two sides of the tracer link 4. That is,

$$
F_{i+3}=c_{0}+\left(b_{4} / a_{4}\right)\left(c_{0}-F_{i}\right), \quad i=1,2,3 .
$$

When both $\Theta_{4}=\Theta_{5}=0$ and any one of the other three links is scaled to zero, the tracing point is at $C_{0}$, indicating that this ground pivot is a triple singular focus. The final case of $\Theta_{4} \neq 0$ and $\Theta_{5} \neq 0$ simultaneously is not possible: it forces $\Theta_{1}=\Theta_{2}=\Theta_{3}=0$, and the four-bar loop cannot be closed. In summary, we have as singular foci the three singular foci of the base four- 


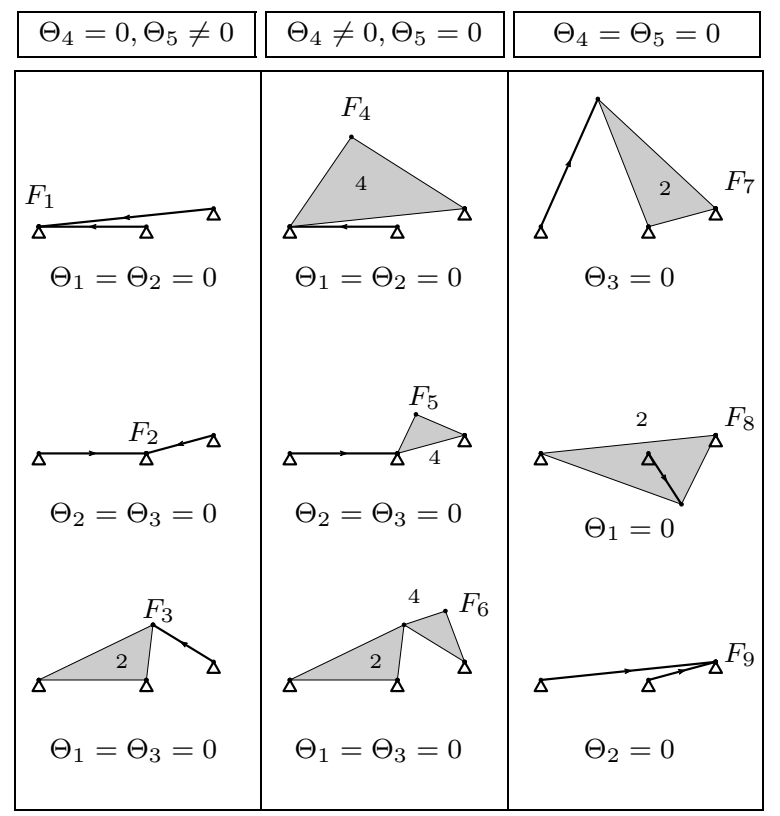

Fig. 4. Focal diagrams for a Stephenson-3 six-bar.

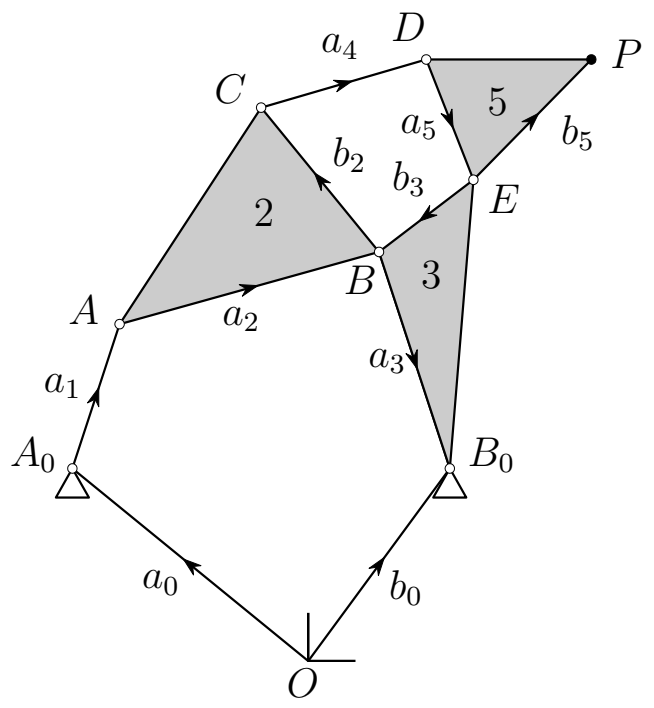

Fig. 5. Watt-1 path-generating linkage

bar, three singular foci that are a stretch-rotation of the four-bar focal triangle, and a triple singular focus at pivot $C_{0}$.

Significantly, the singular foci of the Stephenson-3 depend only on the ground pivots and the shape of links 2 and 4 . The scale of the links does not matter.

As the final example, we examine the Watt-1 linkage drawn in Fig.5. This example requires some extra analysis to deal with solution lines at infinity. This is related to the eigenvalue problem that arises when the method of [17] is applied to this mechanism. 


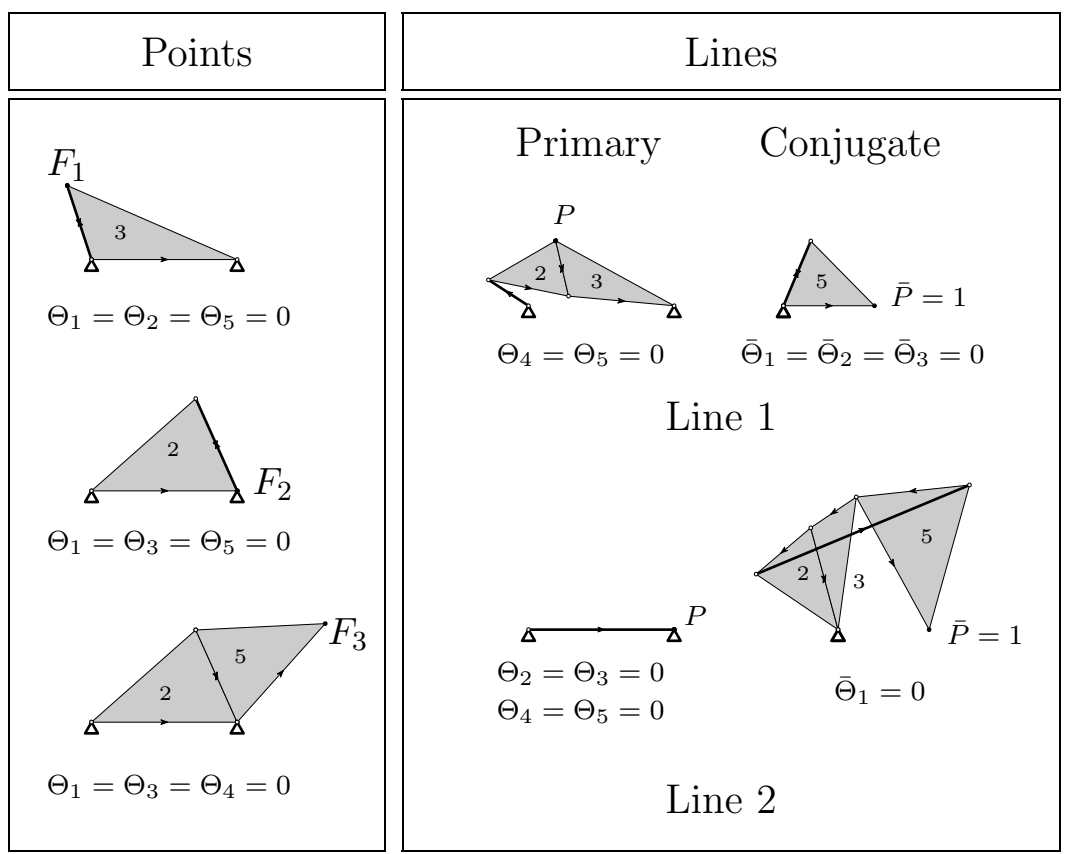

Fig. 6. Solutions at infinity, $(\bar{P}=1, \bar{V}=0)$, for a Watt-1 six-bar.

Example 3 Watt1 focal diagrams. Consider the Watt six-bar linkage shown in Fig.5. Denoting $p=\overrightarrow{O P}$, the loop equations are

$$
\begin{aligned}
\left(a_{0}-b_{0}\right)+a_{1} \theta_{1}+a_{2} \theta_{2}+a_{3} \theta_{3} & =0 \\
b_{2} \theta_{2}+b_{3} \theta_{3}+a_{4} \theta_{4}+a_{5} \theta_{5} & =0 \\
b_{0}-\left(a_{3}+b_{3}\right) \theta_{3}+b_{5} \theta_{5}-p & =0
\end{aligned}
$$

This linkage is more difficult to analyze than the Stephenson-3 previously considered. In the case of the Stephenson-3 linkage, the system of equations (1214) had nine solution points, each giving a focal point. In the case of the Watt-1 linkage, we find that the system has three solution points and two solution lines. These solutions are diagrammed in Fig.6. Expressions for the singular foci are as follows: $F_{1}=a_{0}+\left(a_{0}-b_{0}\right) b_{3} / a_{3}, F_{2}=b_{0}$ and $F_{3}=b_{0}+\left(a_{0}-b_{0}\right) b_{2} b_{5} /\left(a_{2} a_{5}\right)$. Note that singular focus $F_{2}$ is at the ground pivot $B_{0}$.

To understand the line solutions, it helps to write out Eqs.(12-14) explicitly as they apply to this linkage:

$$
\begin{array}{r}
\left(a_{0}-b_{0}\right)+a_{1} \Theta_{1}+a_{2} \Theta_{2}+a_{3} \Theta_{3}=0 \\
b_{2} \Theta_{2}+b_{3} \Theta_{3}+a_{4} \Theta_{4}+a_{5} \Theta_{5}=0 \\
b_{0}-\left(a_{3}+b_{3}\right) \Theta_{3}+b_{5} \Theta_{5}-P=0 \\
a_{1}^{*} \bar{\Theta}_{1}+a_{2}^{*} \bar{\Theta}_{2}+a_{3}^{*} \bar{\Theta}_{3}=0 \\
b_{2}^{*} \bar{\Theta}_{2}+b_{3}^{*} \bar{\Theta}_{3}+a_{4}^{*} \bar{\Theta}_{4}+a_{5}^{*} \bar{\Theta}_{5}=0
\end{array}
$$




$$
\begin{gathered}
\left(a_{3}+b_{3}\right)^{*} \bar{\Theta}_{3}+b_{5}^{*} \bar{\Theta}_{5}-1=0 \\
\Theta_{i} \bar{\Theta}_{i}=0, \quad i=1, \ldots, 5
\end{gathered}
$$

Solution Line 1 in Fig. 6 arises because the first conjugate loop equation, Eq.(20), is satisfied by $\bar{\Theta}_{1}=\bar{\Theta}_{2}=\bar{\Theta}_{3}=0$, which allows all three conjugate loop equations, Eqs.(20-22), to be satisfied with only two conjugate variables, $\bar{\Theta}_{4}$ and $\bar{\Theta}_{5}$, being nonzero. Consequently, in the main loop equations, we have $\Theta_{4}=\Theta_{5}=0$, leaving four variables, $\left\{\Theta_{1}, \Theta_{2}, \Theta_{3}, P\right\}$, to close the three main loop equations, Eqs.(17-19). The point $P$ can be placed anywhere in the plane and still there will be a solution for the remaining variables. However, we are only interested in the points on Line 1 that are limits of the finite tracing curve as it approaches infinity. On the finite curve as it approaches $\Theta_{4}=\Theta_{5}=\bar{V}=0$, we have

$$
\begin{array}{r}
\left(a_{0}-b_{0}\right)+a_{1} \theta_{1}+a_{2} \theta_{2}+a_{3} \theta_{3}=0 \\
b_{2} \theta_{2}+b_{3} \theta_{3}=0 \\
b_{0}-\left(a_{3}+b_{3}\right) \theta_{3}-p=0 \\
\left(a_{0}-b_{0}\right)^{*}+a_{1}^{*} \theta_{1}^{-1}+a_{2}^{*} \theta_{2}^{-1}+a_{3}^{*} \theta_{3}^{-1}=0 .
\end{array}
$$

One may use the second of these to eliminate $\theta_{2}$ from the rest. Then, multiplying the last equation by $\theta_{1} \theta_{3}$ to clear away the negative exponents, one obtains a quadratic equation. This gives two singular foci, shown in Fig. 7. In the conjugate loop diagrams on the right of this figure, links 4 and 5 are infinite in extent. If we zoom out infinitely far, the conjugate diagrams will look just like the one shown in Fig. 6 for Line 1.

Solution Line 2 arises in a complementary fashion to Line 1. This time, the second main loop equation, Eq.(18), is satisfied by $\Theta_{2}=\Theta_{3}=\Theta_{4}=\Theta_{5}=0$, so that only link 1 is needed to bridge across the two ground pivots. This leaves four variables, $\left\{\bar{\Theta}_{2}, \bar{\Theta}_{3}, \bar{\Theta}_{4}, \bar{\Theta}_{5}\right\}$, to close only three loops in the conjugate equations, Eqs.(20-22), thus giving a one-dimensional solution line. Note that Eq.(19) gives $P=b_{0}$, that is, the singular focus remains at the fixed pivot $B_{0}$ along the whole solution line. However, just as for Line 1, we need to determine if Line 2 is approached by the finite portion of the tracing curve. The governing equations in the approach to $\bar{\Theta}_{1}=\bar{V}=0, \bar{P}=1$ are

$$
\begin{array}{r}
a_{2}^{*} \bar{\theta}_{2}+a_{3}^{*} \bar{\theta}_{3}=0 \\
b_{2}^{*} \bar{\theta}_{2}+b_{3}^{*} \bar{\theta}_{3}+a_{4}^{*} \bar{\theta}_{4}+a_{5}^{*} \bar{\theta}_{5}=0 \\
-\left(a_{3}+b_{3}\right)^{*} \bar{\theta}_{3}+b_{5}^{*} \bar{\theta}_{5}-\bar{p}=0 \\
b_{2} \bar{\theta}_{2}^{-1}+b_{3} \bar{\theta}_{3}^{-1}+a_{4} \bar{\theta}_{4}^{-1}+a_{5} \bar{\theta}_{5}^{-1}=0
\end{array}
$$




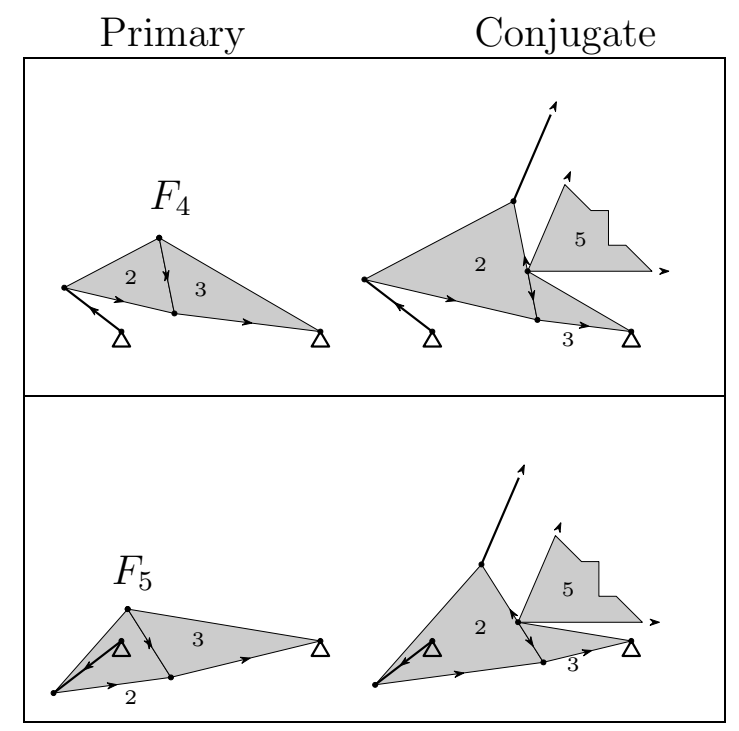

Fig. 7. Focal points on solution Line 1 of Watt-1 six-bar.

Since these are all homogeneous, we may set $\bar{\theta}_{2}=1$ to dehomogenize. Then the first equation determines $\bar{\theta}_{3}=-\left(a_{2} / a_{3}\right)^{*}$. After substituting these into the final equation, we may clear negative exponents by multiplying by $\bar{\theta}_{4} \bar{\theta}_{5}$. We are left with one quadratic equation and two linears, hence there are two solutions. Since the tracing point coincides with fixed pivot $B_{0}$ for every configuration in Line 2 (see Fig.6), we get two more copies of $B_{0}$, which along with focus $F_{2}$ make $B_{0}$ a triple singular focus.

In total, we have $B_{0}$ as a triple singular focus along with four other singular foci. This agrees with the results obtained in [17].

The final observation to make is that Lines 1 and 2 intersect at $\Theta_{2}=\Theta_{3}=$ $\Theta_{4}=\Theta_{5}=\bar{\Theta}_{1}=\bar{\Theta}_{2}=\bar{\Theta}_{3}=0$ with $P=b_{0}$. This degenerate point at infinity is not approached by the finite portion of the tracing curve, so it does not count as a singular focus. However, it appears as the extraneous factor $\left(p-b_{0}\right)\left(\bar{p}-\bar{b}_{0}\right)$ in the Dixon determinant when using the methods of [16,17].

\section{Path Cognates}

A detailed discussion of the use of the singular foci to determine path cognates must be postponed to a later paper. However, we can use the results shown above for the Stephenson-3 six-bar to give a hint of how to proceed.

Two linkages which produce the same tracing curve must have the same singular foci. The Stephenson-3 linkage is drawn in Fig.2, and its singular foci are diagrammed in Fig.4. We see that fixed pivot $C_{0}$ is a triple singular focus and the only multiple focus. Thus, all path cognates must have this point as 
the ground pivot for link 5 . We have already noted that the focal triangles $F_{1} F_{2} F_{3}$ and $F_{4} F_{5} F_{6}$ are similar. For a general Stephenson-3 linkage, no other partitioning of these six foci into two triangles will give similar triangles. Consequently, for any path cognate, one of these must be the focal triangle for the four-bar sub-linkage formed by links $0,1,2,3$, and the other is generated by stretch-rotation about $C_{0}$ via link 4 . We know that the same four-bar curve is generated by three cognates (Robert's cognates), each having the same focal triangle $F_{1} F_{2} F_{3}$, so we immediately get three cognates for the six-bar from this. We can get another three cognates by reversing the roles of the two focal triangles; that is, stretch-rotate the four-bars about $C_{0}$ such that their focal triangle is $F_{4} F_{5} F_{6}$ instead of $F_{1} F_{2} F_{3}$. These are the six path cognates found by Roth [12].

The argument of the last paragraph shows that there are six possible assignments of the singular foci to the fixed pivots of the Stephenson-3 six-bar. Roth's constructions give one path cognate for each of these: the reversal of the roles of the two focal triangles is accomplished via the construction of a Hart pantograph, and reassignment of the fixed pivots within a focal triangle is done via Roberts cognates. To conclude that there are no other cognates, one must show that each assignment of the foci to the fixed pivots determines a unique linkage. This will be done in a future report.

\section{Coupler Mechanisms}

A coupler mechanism consists of two one-degree-of-freedom path-generating mechanisms that are connected by a coupler link, see [14]. This is a special case of the type of planar motion considered in [10]. When a tracing point is placed on the coupler link, the singular foci of the tracing curve of the coupler mechanism are easily derived from those of the two sub-linkages. An example is the Stephenson-3 linkage, where link 4 couples together a four-bar (links $0,1,2,3$ ) with a two-bar (link 5 pinned to link 0 ). We have seen that the singular foci of the six-bar are those of the four-bar $\left(F_{1}, F_{2}, F_{3}\right)$, the center point of the circle generated by link $5\left(C_{0}\right)$, and three obtained by scaling and moving the coupler link to bridge from one of $F_{1}, F_{2}, F_{3}$ to $C_{0}$.

It can be seen from the geometric constructions of $\S 4$ that the pattern of the Stephenson-3 linkage generalizes to any coupler mechanism: its singular foci will be those of its two sub-linkages along with new ones constructed by bridging the coupler link from a singular focus of one sub-linkage to one of the other sub-linkage. If the sub-linkages have $n_{1}$ and $n_{2}$ singular foci, then the bridging gives $n_{1} n_{2}$ new singular foci, for a total of $n_{1}+n_{2}+n_{1} n_{2}$. Those coming directly from the sub-linkages may be multiple singular foci. The multiplicities can be counted by the same construction method, but we will not go into that 
level of detail here.

The special case of "Stephenson-pattern" linkages, which result from sequentially adding any number of output dyads to an initial four-bar, are particularly easy to analyze. Wunderlich [18] found that the coupler curve of the $n$-loop Stephenson-pattern linkage is fully circular of degree $2 \cdot 3^{n}$ (see also $[9$, pp. 227-228]). It thus has $3^{n}$ singular foci. Applying the results of this paper, one can generate all the singular foci recursively. The $n$-loop mechanism inherits $3^{n-1}$ singular foci from the $(n-1)$ loop sub-mechanism it is built on, the ground pivot of the new output dyad is a singular focus of order $3^{n-1}$, and there are $3^{n-1}$ new singular foci obtained by stretch-rotation of the previous foci about the new ground pivot. The stretch-rotation factor is the ratio of two of the sides of the coupler triangle, as we saw in the case of the the Stephenson-3 linkage. The recursion can be started with the "0-loop" linkage, which is a single link pinned to ground. The 0-loop "coupler curve" is just a circle, having degree 2, with a single singular focus at its center. Using the recursive approach just outlined, one can easily determine the 3 singular foci for the 1-loop mechanism, which is just the four-bar linkage, then the 9 singular foci of the 2-loop mechanism, which is the Stephenson-3 six-bar linkage, and so on.

\section{Conclusion}

This paper gives a method for finding the singular foci of planar linkages. Based on analysis of the solutions at infinity of the loop equations, it leads to a detailed geometric interpretation of the foci. A key step in this interpretation is a technique for drawing linkages in non-real configurations. Although the new approach to singular foci must be applied on a case-by-case basis for each linkage type, it yields simple formulae for the singular foci. This is in contrast to the method of [17], which is easier to implement as a general numerical algorithm but does not readily yield simple formulae or geometric understanding. The new method is particularly apt for determining the singular foci of a class of mechanisms called "coupler mechanisms," including all of the Stephenson-pattern linkages.

The singular foci represent essential characteristics of the curve traced out by a planar linkage; in particular, they describe the behavior of the curve at infinity. Two curves sharing one or more singular foci have a reduced number of intersection points and two linkages can generate the same tracing curve only if they have all singular foci in common. These facts can be useful in the design of path-generating linkages. 


\section{References}

[1] R. Bricard, Leons de Cinématique: Tome II, Cinématique Appliquée, GauthierVillars, Paris, 1927.

[2] O. Bottema and B. Roth, Theoretical Kinematics, Dover Publications, New York, 1990.

[3] A. Cayley, "On three-bar motion," Proc. London Math. Soc., Vol. VII, 1875-76, pp. 136-166.

[4] J.L. Coolidge, A Treatise on Algebraic Plane Curves, Dover, New York, 1959.

[5] G. Darboux, "De l'emploi des fonctions elliptiques dans la théorie du quadrilatère plan," Bull. des Sciences Math. Astro., Tome III, 1879.

[6] E. Dijksman, Motion Geometry of Mechanisms, Cambridge University Press, Cambridge, 1976.

[7] A. Haarbleicher, "Application des coordonnées isotropes a l'étude de la courbe des trois barres," J. de l'Ecole Polytechnique, II serie, v. 31, pp. 13-40, 1933.

[8] J. Harris, Algebraic Geometry: A First Course, Springer, New York, 1992.

[9] K.H. Hunt, Kinematic Geometry of Mechanisms, Clarendon Press, Oxford, 1978.

[10] S. Roberts, "On the motion of a plane under certain conditions," Proc. London Math. Soc., Vol. III, pp. 286-319, 1871.

[11] S. Roberts, "On three-bar motion in plane space," Proc. London Math. Soc., Vol. VII, pp. 14-23, 1875.

[12] B. Roth, "On the multiple generation of coupler curves," Trans. ASME, Series B, J. Eng. Industry, v.87, n.2, pp.177-183, 1965.

[13] E.J.F. Primrose, F. Freudenstein and B. Roth, "Six-bar motion (Parts I-III)," Arch. Rational Mech. Anal., v.24, pp.22-77, 1967.

[14] C. Wampler, "Isotropic coordinates, circularity and Bezout numbers: Planar kinematics from a new perspective," Proc. ASME DETC, Aug. 18-22, 1996, Irvine, CA, Paper 96-DETC/MECH-1210.

[15] C. Wampler, "Solving the kinematics of planar mechanisms," ASME J. Mech. Des., v.121, n.3, pp.387-391, 1999.

[16] C. Wampler, "Solving the kinematics of planar mechanisms by Dixon determinant and a complex-plane formulation," ASME J. Mech. Design, v.123, n.3, pp.382-387, 2001.

[17] C. Wampler, "Singular Foci of Planar Linkages," Mech. Mach. Theory (in press).

[18] W. Wunderlich, "Höhere koppelkurven," Öst. Ing. Arch., v.17, n.3, pp.162-165, 1963. 